\title{
Asymptotic Behavior of a Chemostat Model with Stochastic Perturbation on the Dilution Rate
}

\author{
Chaoqun Xu, ${ }^{1}$ Sanling Yuan, ${ }^{1}$ and Tonghua $\mathrm{Zhang}^{2}$ \\ ${ }^{1}$ College of Science, University of Shanghai for Science and Technology, Shanghai 200093, China \\ ${ }^{2}$ Mathematics, FEIS, Swinburne University of Technology, P.O. Box 218 (H38) Hawthorn, VIC 3122, Australia
}

Correspondence should be addressed to Sanling Yuan; sanling@usst.edu.cn

Received 4 October 2012; Revised 1 January 2013; Accepted 4 January 2013

Academic Editor: Ivanka Stamova

Copyright (C) 2013 Chaoqun Xu et al. This is an open access article distributed under the Creative Commons Attribution License, which permits unrestricted use, distribution, and reproduction in any medium, provided the original work is properly cited.

We present a stochastic simple chemostat model in which the dilution rate was influenced by white noise. The long time behavior of the system is studied. Mainly, we show how the solution spirals around the washout equilibrium and the positive equilibrium of deterministic system under different conditions. Furthermore, the sufficient conditions for persistence in the mean of the stochastic system and washout of the microorganism are obtained. Numerical simulations are carried out to support our results.

\section{Introduction}

Modeling microbial growth is a problem of special interest in mathematical biology and theoretical ecology. One particular class of models includes deterministic models of microbial growth in the chemostat. Equations of the basic chemostat model with a single species and a single substrate take the form

$$
\begin{aligned}
& \frac{d S}{d t}=\left(S^{0}-S\right) D-\frac{x}{\gamma} p(S), \\
& \frac{d x}{d t}=x(p(S)-D),
\end{aligned}
$$

where $S(t)$ and $x(t)$, respectively, denote concentrations of the nutrient and the microbial biomass, and all the parameters are positive constants; $S^{0}$ denotes the feed concentration of the nutrient and $D$ denotes the volumetric dilution rate (flow rate/volume). The function $p(S)$ denotes the microbial growth rate and a typical choice for $p(S)$ is $p(S)=(m S) /(a+S)$ [1]. The stoichiometric yield coefficient $\gamma$ denotes the ratio of microbial biomass produced to the mass of the nutrient consumed.

The dynamics of the basic model is simple. If $\gamma$ is constant and $p(S)$ is a monotonically increasing function, then the microorganism can either become extinct or persist at an equilibrium level [2-5]. The particular outcome depends solely on the break-even concentration $\lambda$ where $p(\lambda)=D$.
Specifically, if $\lambda<S^{0}$, the organism persists, and if $\lambda \geq S^{0}$, it becomes extinct.

Note that the modeling process that leads to (1) relies on the fact that the stochastic effects can be neglected or averaged out, thanks to the law of large numbers. This is possible only at macroscopic scale, for large population sizes, and under homogeneity conditions. At all other scales or when the homogeneity conditions are not met, random effects cannot be neglected, just as that stated in Campillo et al. [6]: "This is the case at microscopic scale, in small population size, as well as all scales preceding the one where (1) is valid. This is also when the homogeneity condition is not met, for example, in unstirred conditions. Also the accumulation of small perturbations in the context of multispecies could not be neglected. Moreover, whereas the experimental results observed in well mastered laboratory conditions match closely the ODE theoretical behavior, a noticeable difference may occur in operational." So, even if the description (1) is sufficient for a number of applications of interest, it does not account for the stochastic aspects of the problem (see Campillo et al. [6] for more details on this respect).

In fact, ecosystem dynamics is inevitably affected by environmental white noise which is an important component in realism. It is therefore useful to reveal how the noise affects the ecosystem dynamics. As a matter of fact, stochastic biological systems and stochastic epidemic models have recently been studied by many authors; see [7-16]. We also 
refer the readers to Imhof and Walcher [7] for the reason why the stochastic effects should be considered in chemostat modeling.

Taking into account the effect of randomly fluctuating environment, we introduce randomness into model (1) by replacing the dilution rate $D$ by $D \rightarrow D+\alpha \dot{B}(t)$, where $\dot{B}(t)$ is a white noise (i.e., $B(t)$ is a Brownian motion) and $\alpha \geq 0$ represents the intensity of noise. This is only a first step in introducing stochasticity into the model. Ideally we would also like to introduce stochastic environmental variation into the other parameters such as the feed nutrient concentration $S^{0}$ and the microbial growth rate $p(S)$, but to do this would make the analysis too difficult. As a result, model (1) becomes the following system of stochastic differential equation:

$$
\begin{gathered}
d S=\left[\left(S^{0}-S\right) D-\frac{x}{\gamma} p(S)\right] d t+\alpha\left(S^{0}-S\right) d B(t), \\
d x=x(p(S)-D) d t-\alpha x d B(t) .
\end{gathered}
$$

To the best of our knowledge, a very little amount of work has been done with the above model. Motivated by this, in this paper, we will investigate the long time behavior of model (2) with $p(S)$ taking the Holling II functional response function, that is, $p(S)=(m S) /(a+S)$.

We should point out that a few papers have already addressed the stochastic modeling of the chemostat $[6,7,17-$ 19]. Here we only mention a recent paper by Imhof and Walcher [7]. They introduced a variant of the deterministic single-substrate chemostat model for which the persistence of all species is possible. To derive a stochastic model they considered a discrete-time Markov process with jumps corresponding to the deterministic system added with a centered Gaussian term, letting the time step converges to zero leads to a system of stochastic differential equations. They proved that random effects may lead to extinction in scenarios where the deterministic model predicts persistence; they also established some stochastic persistence results. Obviously, the model they considered is different from our model (2). We also refer the readers to Campillo et al. [6] for other works on the stochastic modeling of the chemostat.

The organization of this paper is as follows. The model and some basic results about the model are presented in the next section. In Section 3, the long time behavior of the stochastic model, including the asymptotic behavior around the positive equilibrium point and that around the extinction equilibrium point, is analyzed. An extinction result on the model is presented in Section 4. Finally, in Section 5, simulations and discussions are presented.

\section{The Model and Some Fundamental Results}

Assuming in model (2) that $p(S)$ takes the Holling II functional response function, that is, $p(S)=(m S) /(a+S)$, consider the following stochastic chemostat model:

$$
\begin{gathered}
d S=\left[\left(S^{0}-S\right) D-\frac{m S x}{a+S}\right] d t+\alpha\left(S^{0}-S\right) d B(t), \\
d x=x\left(\frac{m S}{a+S}-D\right) d t-\alpha x d B(t),
\end{gathered}
$$

where $S(t)$ and $x(t)$ have the similar biological meanings as in model (1), and all parameters are positive constants. $S^{0}$, $D$, and $\alpha$ play similar roles as in model (2), and $m$ and $a$ are the maximal growth rates of the organism and the MichaelisMenten (or half saturation) constant, respectively; the yield coefficient $\gamma$ has been scaled out by scaling.

As $S(t)$ and $x(t)$ in (3) are concentrations of the substrate and the microorganism at time $t$, respectively, we are only interested in the positive solutions. Moreover, in order for a stochastic differential equation to have a unique global (i.e., no explosion in a finite time) solution for any given initial value, the coefficients of the equation are generally required to satisfy the linear growth condition and local Lipschitz condition. However, the coefficients of (3) do not satisfy the linear growth condition, though they are locally Lipschitz continuous, so the solution of model (3) may explode at a finite time [20,21]. In the following, by using the comparison theorem for stochastic equation (see $[8,22]$ ), we first show the solution of model (3) is positive and global.

Theorem 1. For any given initial value $\left(S_{0}, x_{0}\right) \in \mathbb{R}_{+}^{2}$, there is a unique positive solution $(S(t), x(t))$ to model (3) on $t \geq 0$, and the solution will remain in $\mathbb{R}_{+}^{2}$ with probability one, namely, $(S(t), x(t)) \in \mathbb{R}_{+}^{2}$ for all $t \geq 0$ almost surely.

Proof. Since the coefficients of model (3) are locally Lipschitz continuous for any given initial value $\left(S_{0}, x_{0}\right) \in \mathbb{R}_{+}^{2}$, there is a unique positive local solution $(S(t), x(t))$ on $t \in\left[0, \tau_{e}\right)$, where $\tau_{e}$ is the explosion time (i.e., the moment at which the solution tends to infinity). Next, we show that $\tau_{e}=\infty$.

Since the solution is positive, we have

$$
\begin{aligned}
& d S \leq\left(S^{0}-S\right)(D d t+\alpha d B(t)), \\
& d x \leq x(m-D) d t-\alpha x d B(t) .
\end{aligned}
$$

Denote by $\Phi(t)$ the solution of the following stochastic differential equation:

$$
\begin{gathered}
d \Phi=\left(S^{0}-\Phi\right)(D d t+\alpha d B(t)), \\
\Phi(0)=S_{0},
\end{gathered}
$$

and $\Psi(t)$ the solution of the equation

$$
\begin{gathered}
d \Psi=(m-D) \Psi d t-\alpha \Psi d B(t), \\
\Psi(0)=x_{0} .
\end{gathered}
$$

By the comparison theorem for stochastic equations, we have $S(t) \leq \Phi(t), x(t) \leq \Psi(t)$, and $t \in\left[0, \tau_{e}\right)$, a.s.

Similarly, we can get

$$
\begin{gathered}
d S \geq\left[\left(S^{0}-S\right) D-m \Psi\right] d t+\alpha\left(S^{0}-S\right) d B(t), \\
d x \geq-x(D d t+\alpha d B(t)) .
\end{gathered}
$$

Denote by $\varphi$ the solution of stochastic differential equation

$$
\begin{gathered}
d \varphi=\left[\left(S^{0}-\varphi\right) D-m \Psi\right] d t+\alpha\left(S^{0}-\varphi\right) d B(t), \\
\varphi(0)=S_{0},
\end{gathered}
$$


and $\psi$ the solution of the equation

$$
\begin{gathered}
d \psi=-\psi(D d t+\alpha d B(t)), \\
\psi(0)=x_{0} .
\end{gathered}
$$

We have that $S(t) \geq \varphi(t), x(t) \geq \psi(t), t \in\left[0, \tau_{e}\right)$, a.s.

To sum up, we have that

$$
\begin{aligned}
& \varphi(t) \leq S(t) \leq \Phi(t), \quad t \in\left[0, \tau_{e}\right), \text { a.s. } \\
& \psi(t) \leq x(t) \leq \Psi(t),
\end{aligned}
$$

Noting that (5)-(9) are all linear stochastic differential equations, $\Phi(t), \Psi(t), \varphi(t)$, and $\psi(t)$ can be explicitly solved from them, separately. Obviously, they are all positive and globally existent for all $t \in[0, \infty)$. From (10), we can have that $\tau_{e}=\infty$. The proof is thus completed.

The following theorem shows that the solution $(S(t), x(t))$ of model (3) with any positive initial value is uniformly bounded in mean.

Theorem 2. For any given initial value $\left(S_{0}, x_{0}\right) \in \mathbb{R}_{+}^{2}$, the solution $(S(t), x(t))$ of model (3) has the property:

$$
\lim _{t \rightarrow \infty} E(S(t)+x(t))=S^{0}
$$

Proof. Define the function $V(t)=S(t)+x(t)$, by the Itô formula, we get

$$
d V=\left(S^{0}-V(S, x)\right)(D d t+\alpha d B) .
$$

Integrating both sides from 0 to $t$, and then taking expectations, yields

$$
E V(t)=V(0)+\int_{0}^{t}\left(s^{0}-E V(s)\right) D d s
$$

Consequently,

$$
\frac{d E V(t)}{d t}=\left(S^{0}-E V(t)\right) D
$$

It is clear that

$$
\lim _{t \rightarrow \infty} E V(t)=S^{0}
$$

Thus, we complete the proof of Theorem 2.

\section{The Long Time Behavior of Model (3)}

When $\alpha=0$, model (3) becomes its corresponding deterministic system

$$
\begin{gathered}
d S=\left[\left(S^{0}-S\right) D-\frac{m S x}{a+S}\right] d t, \\
d x=x\left(\frac{m S}{a+S}-D\right) d t .
\end{gathered}
$$

We have known that the dynamic behavior of model (16) is completely determined by the break-even concentration
$\lambda=D a /(m-D)$. Model (16) always has a washout equilibrium $E^{0}=\left(S^{0}, 0\right)$. When $\lambda \geq S^{0}, E^{0}$ is globally asymptotically stable, and when $0<\lambda<S^{0}, E^{0}$ loses its stability and a globally asymptotically stable positive equilibrium $E^{*}=$ $\left(S^{*}, x^{*}\right)$ appears, where $S^{*}=\lambda$ and $x^{*}=S^{0}-\lambda$.

When $\alpha \neq 0, E^{0}$ is still an equilibrium of model (3) but $E^{*}$ is not; that is to say, model (3) has no positive equilibrium. It is natural to ask whether the microorganism will persist or go to extinction in the chemostat. In this section we mainly use the way of estimating the oscillation around $E^{*}$ (or $E^{0}$ ) to reflect how the solution of model (3) spirals around $E^{*}$ (or $\left.E^{0}\right)$. In the following part of this section, we always denote $\delta_{0}$ by

$$
\delta_{0}=D-\frac{\alpha^{2}}{2}
$$

3.1. Asymptotic Behavior around the Washout Equilibrium $E^{0}$. As mentioned above, system (16) always has an equilibrium $E^{0}$ which is globally asymptotically stable provided $\lambda \geq S^{0}$. It is natural to ask whether the solutions of system (3) will be close to $E^{0}$. We have the following theorem.

Theorem 3. If $\lambda \geq S^{0}$ and $\delta_{0}>0$, then the washout equilibrium $E^{0}$ of system (3) is stochastically asymptotically stable in the large.

Proof. Define a function $V: \mathbb{R}_{+}^{2} \rightarrow \mathbb{R}_{+}$by

$$
V(S, x)=\left(S+x-S^{0}\right)^{2}+\frac{4 \delta_{0} a}{m} x .
$$

Obviously, the function $V$ is positive definite, and

$$
d V=L V d t-\alpha\left[2\left(S+x-S^{0}\right)^{2}+\frac{4 \delta_{0} a}{m} x\right] d B
$$

where,

$$
\begin{aligned}
L V= & -2 D\left(S+x-S^{0}\right)^{2}+\alpha^{2}\left(S^{0}-S\right)^{2}-2 \alpha^{2}\left(S^{0}-S\right) x \\
& +\alpha^{2} x^{2}+\frac{4 \delta_{0} a}{m}\left(\frac{m S}{a+S}-D\right) x \\
= & -2 \delta_{0}\left(S+x-S^{0}\right)^{2}+\frac{4 \delta_{0} a}{m}\left(\frac{m S}{a+S}-D\right) x \\
= & -2 \delta_{0}\left[\left(S-S^{0}\right)^{2}+x^{2}\right]+\frac{4 \delta_{0} a}{m}\left(\frac{m S}{a+S}-D\right) x \\
& -4 \delta_{0}\left(S-S^{0}\right) x .
\end{aligned}
$$

We will show that for all solutions $(S(t), x(t))$ of system (3) with initial value $\left(S_{0}, x_{0}\right) \in \mathbb{R}_{+}^{2}$,

$$
L V \leq-\frac{2 \delta_{0} a^{2}}{\left(a+S^{0}\right)^{2}}\left[\left(S-S^{0}\right)^{2}+x^{2}\right]
$$


Suppose first that $\lambda \geq S^{0}$, then

$$
\frac{m S}{a+S}-D \leq \frac{m S}{a+S}-\frac{m S^{0}}{a+S^{0}} \leq \frac{m}{a}\left(S-S^{0}\right) .
$$

Obviously,

$$
\frac{4 \delta_{0} a}{m}\left(\frac{m S}{a+S}-D\right) x-4 \delta_{0}\left(S-S^{0}\right) x \leq 0 .
$$

It then follows from (20) that

$$
L V \leq-2 \delta_{0}\left[\left(S-S^{0}\right)^{2}+x^{2}\right] .
$$

Suppose next that $S \leq S^{0}$; noting also that $\lambda \geq S^{0}$, we have

$$
\frac{m S}{a+S}-D \leq \frac{m S}{a+S}-\frac{m S^{0}}{a+S^{0}} \leq \frac{m a}{\left(a+S^{0}\right)^{2}}\left(S^{0}-S\right) .
$$

Therefore,

$$
\begin{aligned}
& \frac{4 \delta_{0} a}{m}\left(\frac{m S}{a+S}-D\right) x-4 \delta_{0}\left(S-S^{0}\right) x \\
& \quad \leq 4 \delta_{0}\left[1-\frac{a^{2}}{\left(a+S^{0}\right)^{2}}\right]\left(S^{0}-S\right) x \\
& \quad \leq 2 \delta_{0}\left[1-\frac{a^{2}}{\left(a+S^{0}\right)^{2}}\right]\left[\left(S-S^{0}\right)^{2}+x^{2}\right] .
\end{aligned}
$$

It follows from (20) that

$$
L V \leq-\frac{2 \delta_{0} a^{2}}{\left(a+S^{0}\right)^{2}}\left[\left(S-S^{0}\right)^{2}+x^{2}\right] .
$$

To sum up, (21) holds. That is to say, $L V$ is negative definite. By Theorem $A$ in the appendix, the washout equilibrium $E^{0}$ of system (3) is stochastically asymptotically stable in the large. The proof of Theorem 3 is thus completed.

\subsection{Asymptotic Behavior around the Positive Equilibrium $E^{*}$}

Theorem 4. If $0<\lambda<S^{0}$ and $\delta_{0}>0$, then for any solution $(S(t), x(t))$ of system (3) with initial value $\left(S_{0}, x_{0}\right) \in \mathbb{R}_{+}^{2}$,

$$
\begin{gathered}
\limsup _{t \rightarrow \infty} \frac{1}{t} \int_{0}^{t}\left[\left(S(s)-S^{*}\right)^{2}+\left(x(s)-x^{*}\right)^{2}\right] d s \\
\leq \frac{2\left(a+S^{*}+4 x^{*}\right)^{2} x^{*}}{m a} \alpha^{2} .
\end{gathered}
$$

Proof. The proof is inspired by the method of Imhof and Walcher [7]. Define a function $V: \mathbb{R}_{+}^{2} \rightarrow \mathbb{R}_{+}$by

$$
V(S, x)=\left(S+x-S^{0}\right)^{2}+\frac{4 \delta_{0} a}{m}\left(x-x^{*}-x^{*} \ln \frac{x}{x^{*}}\right) .
$$

Obviously, the function $V$ is positive definite. Using Itô's formula we get

$$
d V=L V d t-2 \alpha\left[\left(S+x-S^{0}\right)^{2}+\frac{2 \delta_{0} a}{m}\left(x-x^{*}\right)\right] d B,
$$

where

$$
\begin{aligned}
L V= & -2 \delta_{0}\left(S+x-S^{0}\right)^{2} \\
& +\frac{4 \delta_{0} a}{m}\left(\frac{m S}{a+S}-D\right)\left(x-x^{*}\right)+\frac{2 \delta_{0} a}{m} \alpha^{2} x^{*} \\
= & -2 \delta_{0}\left[\left(S-S^{*}\right)^{2}+\left(x-x^{*}\right)^{2}\right]+\frac{2 \delta_{0} a}{m} \alpha^{2} x^{*} \\
& +\frac{4 \delta_{0} a}{m}\left(\frac{m S}{a+S}-D\right)\left(x-x^{*}\right) \\
& -4 \delta_{0}\left(S-S^{*}\right)\left(x-x^{*}\right) .
\end{aligned}
$$

We will show that for all solutions $(S(t), x(t))$ with initial value $\left(S_{0}, x_{0}\right) \in \mathbb{R}_{+}^{2}$,

$$
\begin{aligned}
L V \leq & -\frac{\delta_{0} a^{2}}{\left(a+S^{*}+4 x^{*}\right)^{2}}\left[\left(S-S^{*}\right)^{2}+\left(x-x^{*}\right)^{2}\right] \\
& +\frac{2 \delta_{0} a}{m} \alpha^{2} x^{*} .
\end{aligned}
$$

Suppose first that $\left(S-S^{*}\right)\left(x-x^{*}\right) \geq 0$, then $(m S /(a+S)-$ $D)\left(x-x^{*}\right) \geq 0$ and

$$
\begin{aligned}
\left(\frac{m S}{a+S}-D\right)\left(x-x^{*}\right) & =\left|\frac{m S}{a+S}-D\right|\left|x-x^{*}\right| \\
& \leq \frac{m}{a}\left|S-S^{*}\right|\left|x-x^{*}\right| \\
& =\frac{m}{a}\left(S-S^{*}\right)\left(x-x^{*}\right) .
\end{aligned}
$$

Obviously,

$$
\frac{4 \delta_{0} a}{m}\left(\frac{m S}{a+S}-D\right)\left(x-x^{*}\right)-4 \delta_{0}\left(S-S^{*}\right)\left(x-x^{*}\right) \leq 0 .
$$

By (31) we have

$$
L V \leq-2 \delta_{0}\left[\left(S-S^{*}\right)^{2}+\left(x-x^{*}\right)^{2}\right]+\frac{2 \delta_{0} a}{m} \alpha^{2} x^{*} .
$$

Suppose next that $\left(S-S^{*}\right)\left(x-x^{*}\right)<0$ and $S>S^{*}+4 x^{*}$ (which implies that $S-S^{*}>0, x-x^{*}<0$ ), then

$$
\begin{aligned}
-4 \delta_{0}\left(S-S^{*}\right)\left(x-x^{*}\right) & =4 \delta_{0}\left(S-S^{*}\right)\left(x^{*}-x\right) \\
& \leq 4 \delta_{0}\left(S-S^{*}\right) x^{*} \\
& \leq \delta_{0}\left(S-S^{*}\right)^{2} .
\end{aligned}
$$

By (31), we have

$$
\begin{aligned}
L V & \leq-\delta_{0}\left(S-S^{*}\right)^{2}-2 \delta_{0}\left(x-x^{*}\right)^{2}+\frac{2 \delta_{0} a}{m} \alpha^{2} x^{*} \\
& \leq-\delta_{0}\left[\left(S-S^{*}\right)^{2}+\left(x-x^{*}\right)^{2}\right]+\frac{2 \delta_{0} a}{m} \alpha^{2} x^{*} .
\end{aligned}
$$


Finally, if $\left(S-S^{*}\right)\left(x-x^{*}\right)<0$ and $S \leq S^{*}+4 x^{*}$, then

$$
\begin{aligned}
& \frac{4 \delta_{0} a}{m}\left(\frac{m S}{a+S}-D\right)\left(x-x^{*}\right)-4 \delta_{0}\left(S-S^{*}\right)\left(x-x^{*}\right) \\
& \quad=4 \delta_{0}\left|S-S^{*}\right|\left|x-x^{*}\right|-\frac{4 \delta_{0} a}{m}\left|\frac{m S}{a+S}-D\right|\left|x-x^{*}\right| \\
& \leq 4 \delta_{0}\left|S-S^{*}\right|\left|x-x^{*}\right|-\frac{4 \delta_{0} a^{2}}{\left(a+S^{*}+4 x^{*}\right)^{2}}\left|S-S^{*}\right|\left|x-x^{*}\right| \\
& =4 \delta_{0}\left[1-\frac{a^{2}}{\left(a+S^{*}+4 x^{*}\right)^{2}}\right]\left|S-S^{*}\right|\left|x-x^{*}\right| \\
& \leq 2 \delta_{0}\left[1-\frac{a^{2}}{\left(a+S^{*}+4 x^{*}\right)^{2}}\right]\left[\left(S-S^{*}\right)^{2}+\left(x-x^{*}\right)^{2}\right] .
\end{aligned}
$$

By (31) we have

$$
\begin{aligned}
L V \leq & -\frac{2 \delta_{0} a^{2}}{\left(a+S^{*}+4 x^{*}\right)^{2}}\left[\left(S-S^{*}\right)^{2}+\left(x-x^{*}\right)^{2}\right] \\
& +\frac{2 \delta_{0} a}{m} \alpha^{2} x^{*} .
\end{aligned}
$$

To sum up, (32) holds. Therefore,

$$
\begin{aligned}
d \mathrm{~V} \leq & \left\{-\frac{\delta_{0} a^{2}}{\left(a+S^{*}+4 x^{*}\right)^{2}}\left[\left(S-S^{*}\right)^{2}+\left(x-x^{*}\right)^{2}\right]\right. \\
& \left.+\frac{2 \delta_{0} a}{m} \alpha^{2} x^{*}\right\} d t \\
& -2 \alpha\left[\left(S+x-S^{0}\right)^{2}+\frac{2 \delta_{0} a}{m}\left(x-x^{*}\right)\right] d B .
\end{aligned}
$$

Integrating both sides from 0 to $t$ yields

$$
\begin{aligned}
V(t)-V(0) \leq \int_{0}^{t}\{ & -\frac{\delta_{0} a^{2}}{\left(a+S^{*}+4 x^{*}\right)^{2}} \\
& \times\left[\left(S(s)-S^{*}\right)^{2}+\left(x(s)-x^{*}\right)^{2}\right] \\
& \left.+\frac{2 \delta_{0} a}{m} \alpha^{2} x^{*}\right\} d s+M(t),
\end{aligned}
$$

where $M(t)=-2 \alpha \int_{0}^{t}\left[\left(S(s)+x-S^{0}\right)^{2}+\left(2 \delta_{0} a / m\right)\left(x(s)-x^{*}\right)\right] d B$. Obviously, $M(t)$ is a local continuous martingale. From (3), we can get

$$
\begin{aligned}
d & \left(S+x-S^{0}\right) \\
& =\left(S^{0}-S-x\right) D d t+\left(S^{0}-S-x\right) \alpha d B \\
& =\left(S+x-S^{0}\right)(-D d t-\alpha d B) .
\end{aligned}
$$

It follows that

$$
\begin{aligned}
S+x-S^{0} & =\left(S_{0}+x_{0}-S^{0}\right) \exp \left\{\left(-D-\frac{\alpha^{2}}{2}\right) t-\alpha B(t)\right\} \\
& \leq C \exp \{-\alpha B(t)\},
\end{aligned}
$$

where $C=\left|S_{0}+x_{0}-S^{0}\right|$. Thus we have that

$$
\begin{aligned}
& \langle M(t), M(t)\rangle_{t} \\
& =4 \alpha^{2} \int_{0}^{t}\left[\left(S(s)+x(s)-S^{0}\right)^{2}+\frac{2 \delta_{0} a}{m}\left(x(s)-x^{*}\right)\right]^{2} d s \\
& \leq 4 \alpha^{2} \int_{0}^{t}\left[C^{2} \exp \left\{-2 \alpha \min _{0 \leq s \leq t} B(s)\right\}+\frac{2 \delta_{0} a}{m}\right. \\
& \left.\times\left(x^{*}+S^{0}+C \exp \left\{-\alpha \min _{0 \leq s \leq t} B(s)\right\}\right)\right]^{2} d s,
\end{aligned}
$$

which implies that

$$
\limsup _{t \rightarrow \infty} \frac{\langle M(t), M(t)\rangle_{t}}{t}<\infty
$$

By Strong Law of Large Numbers (see Mao [21]), we obtain

$$
\lim _{t \rightarrow \infty} \frac{M(t)}{t}=0 \text { a.s. }
$$

It follows from (41) that

$$
\begin{aligned}
\limsup _{t \rightarrow \infty} \frac{1}{t} \int_{0}^{t}\{ & -\frac{\delta_{0} a^{2}}{\left(a+S^{*}+4 x^{*}\right)^{2}} \\
& \times\left[\left(S(s)-S^{*}\right)^{2}+\left(x(s)-x^{*}\right)^{2}\right] \\
& \left.+\frac{2 \delta_{0} a}{m} \alpha^{2} x^{*}\right\} d s \geq 0,
\end{aligned}
$$

namely,

$$
\begin{gathered}
\limsup _{t \rightarrow \infty} \frac{1}{t} \int_{0}^{t}\left[\left(S(s)-S^{*}\right)^{2}+\left(x(s)-x^{*}\right)^{2}\right] d s \\
\leq \frac{2\left(a+S^{*}+4 x^{*}\right)^{2} x^{*}}{a m} \alpha^{2} .
\end{gathered}
$$

This completes the proof of Theorem 4 .

Remark 5. Theorems 3 and 4 show that the solutions of system (3) will fluctuate, respectively, around $E^{0}$ and $E^{*}$ when $\alpha$ is small enough such that $\delta_{0}>0$ (i.e., $\alpha<\sqrt{2 D}$ ).

3.3. Persistence of System (3). From the result of Theorem 4, we conclude that system (3) is persistent when $\alpha$ is small, which implies the persistence of microorganism. L. S. Chen and J. Chen in [23] proposed the definition of persistence in the mean for the deterministic system. Here, we also use this definition for the stochastic system (see also Ji et al. [24]).

Definition 6. System (3) is said to be persistent in the mean, if

$$
\liminf _{t \rightarrow \infty} \frac{1}{t} \int_{0}^{t} S(s) d s>0, \quad \liminf _{t \rightarrow \infty} \frac{1}{t} \int_{0}^{t} x(s) d s>0 \text { a.s. }
$$


Theorem 7. Let $(S(t), x(t))$ be the solution of system (3) with initial value $\left(S_{0}, x_{0}\right) \in \mathbb{R}_{+}^{2}$. If $0<\lambda<S^{0}, \delta_{0}>0$, and

$$
\alpha^{2}<\min \left\{\frac{a m\left(S^{*}\right)^{2}}{2\left(a+S^{*}+4 x^{*}\right)^{2} x^{*}}, \frac{a m x^{*}}{2\left(a+S^{*}+4 x^{*}\right)^{2}}\right\},
$$

then system (3) is persistent in the mean.

Proof. Obviously, (28) holds. It follows that

$$
\begin{aligned}
& \limsup _{t \rightarrow \infty} \frac{1}{t} \int_{0}^{t}\left(S(s)-S^{*}\right)^{2} d s \leq \frac{2\left(a+S^{*}+4 x^{*}\right)^{2} x^{*}}{a m} \alpha^{2} \text { a.s., } \\
& \limsup _{t \rightarrow \infty} \frac{1}{t} \int_{0}^{t}\left(x(s)-x^{*}\right)^{2} d s \leq \frac{2\left(a+S^{*}+4 x^{*}\right)^{2} x^{*}}{a m} \alpha^{2} \text { a.s. }
\end{aligned}
$$

Noting also that

$$
2\left(S^{*}\right)^{2}-2 S^{*} S=2 S^{*}\left(S^{*}-S\right) \leq\left(S^{*}\right)^{2}+\left(S-S^{*}\right)^{2},
$$

we have

$$
S \geq \frac{S^{*}}{2}-\frac{\left(S-S^{*}\right)^{2}}{2 S^{*}} .
$$

It follows from (54), (50), and (51) that

$$
\begin{aligned}
\liminf _{t \rightarrow \infty} \frac{1}{t} \int_{0}^{t} S(s) d s \\
\geq \frac{S^{*}}{2}-\limsup _{t \rightarrow \infty} \frac{1}{t} \int_{0}^{t} \frac{\left(S(s)-S^{*}\right)^{2}}{2 S^{*}} d s \\
\geq \frac{S^{*}}{2}-\frac{2\left(a+S^{*}+4 x^{*}\right)^{2} x^{*}}{2 a m S^{*}} \alpha^{2}>0 \text { a.s. }
\end{aligned}
$$

Similarly, we have

$$
\begin{aligned}
\liminf _{t \rightarrow \infty} \frac{1}{t} \int_{0}^{t} x(s) d s \\
\geq \frac{x^{*}}{2}-\limsup _{t \rightarrow \infty} \frac{1}{t} \int_{0}^{t} \frac{\left(x(s)-x^{*}\right)^{2}}{2 x^{*}} d s \\
\geq \frac{x^{*}}{2}-\frac{2\left(a+S^{*}+4 x^{*}\right)^{2} x^{*}}{2 a m x^{*}} \alpha^{2}>0 \text { a.s. }
\end{aligned}
$$

Therefore, system (3) is persistent in the mean.

\section{Washout of the Organism in the Chemostat}

The following theorem shows that sufficiently large noise can make the microorganism extinct exponentially with probability one in a simple chemostat.

Theorem 8. For any given initial value $\left(S_{0}, x_{0}\right) \in \mathbb{R}_{+}^{2}$, the solution $(S(t), x(t))$ of system (3) has the property:

$$
\limsup _{t \rightarrow \infty} \frac{\ln x(t)}{t} \leq(m-D)-\frac{1}{2} \alpha^{2} .
$$

Proof. Define the function $V(x)=\ln x$; by the Itô formula, we get

$$
\begin{aligned}
d V & =\frac{1}{x} d x-\frac{1}{2 x^{2}}(d x)^{2} \\
& =\left(\frac{m S}{a+S}-D\right) d t-\alpha d B-\frac{1}{2} \alpha^{2} d t \\
& \leq\left(m-D-\frac{1}{2} \alpha^{2}\right) d t-\alpha d B .
\end{aligned}
$$

Integrating both sides from 0 to $t$ yields

$$
\ln x(t)-\ln x(0) \leq\left(m-D-\frac{1}{2} \alpha^{2}\right) t-\alpha B(t) .
$$

Dividing $t$ on the both sides and letting $t \rightarrow \infty$, we have

$$
\limsup _{t \rightarrow \infty} \frac{\ln x(t)}{t} \leq(m-D)-\frac{1}{2} \alpha^{2} .
$$

The proof of Theorem 8 is completed.

Remark 9. In fact, we can see from the proof of Theorem 8 that only the boundedness of $p(S)=m S /(a+S)$ is used. So, Theorem 8 holds true for a much larger class bounded growth rate functions, for example, $p(S)=m S /\left(a+b S+S^{2}\right)$ (see also Figure 4).

\section{Simulations and Discussions}

In order to confirm the results above, we numerically simulate the solution of system (3) and system (16) with the initial $\left(S_{0}, x_{0}\right)=(0.7,0.3)$. The numerical simulation is given by the following Milstein scheme [25]. Consider the discretization of system (3) for $t=0, \Delta t, 2 \Delta t, \ldots, n \Delta t$ :

$$
\begin{aligned}
S_{i+1}= & S_{i}+\left[\left(S^{0}-S_{i}\right) D-\frac{m S_{i} x_{i}}{a+S_{i}}\right] \Delta t \\
& +\alpha\left(S^{0}-S_{i}\right) \sqrt{\Delta t} \xi_{i}, \\
x_{i+1}= & x_{i}+x_{i}\left(\frac{m S_{i}}{a+S_{i}}-D\right) \Delta t-\alpha x_{i} \sqrt{\Delta t} \xi_{i},
\end{aligned}
$$

where time increment $\Delta t>0$, and $\xi_{\mathrm{i}}$ is $N(0,1)$-distributed independent random variables which can be generated numerically by pseudorandom number generators. In Figures 1-4, we will use the blue lines and the red lines to represent the solutions of deterministic system (16) and those of stochastic system (3), respectively.

By Theorem 3, we expect that the washout equilibrium $E^{0}$ of system (3) is globally asymptotically stable under the conditions $\lambda \geq S^{0}$ and $\delta_{0}>0$. In Figure 1, we choose parameters $S^{0}=1, m=2, a=0.6, D=1.3$, and $\alpha=0.2$ in Figure 1(a). We can compute that $\lambda=39 / 35>1=S^{0}$ and $\delta_{0}=1.28>0$. Then the washout equilibrium $E^{0}$ of systems (3) and (16) is globally asymptotically stable (see Figure 1(a)). Furthermore, to watch the influence of the intensity of noise on the dynamics of system (3), we take $\alpha=0.5$ in Figure 1(b). 

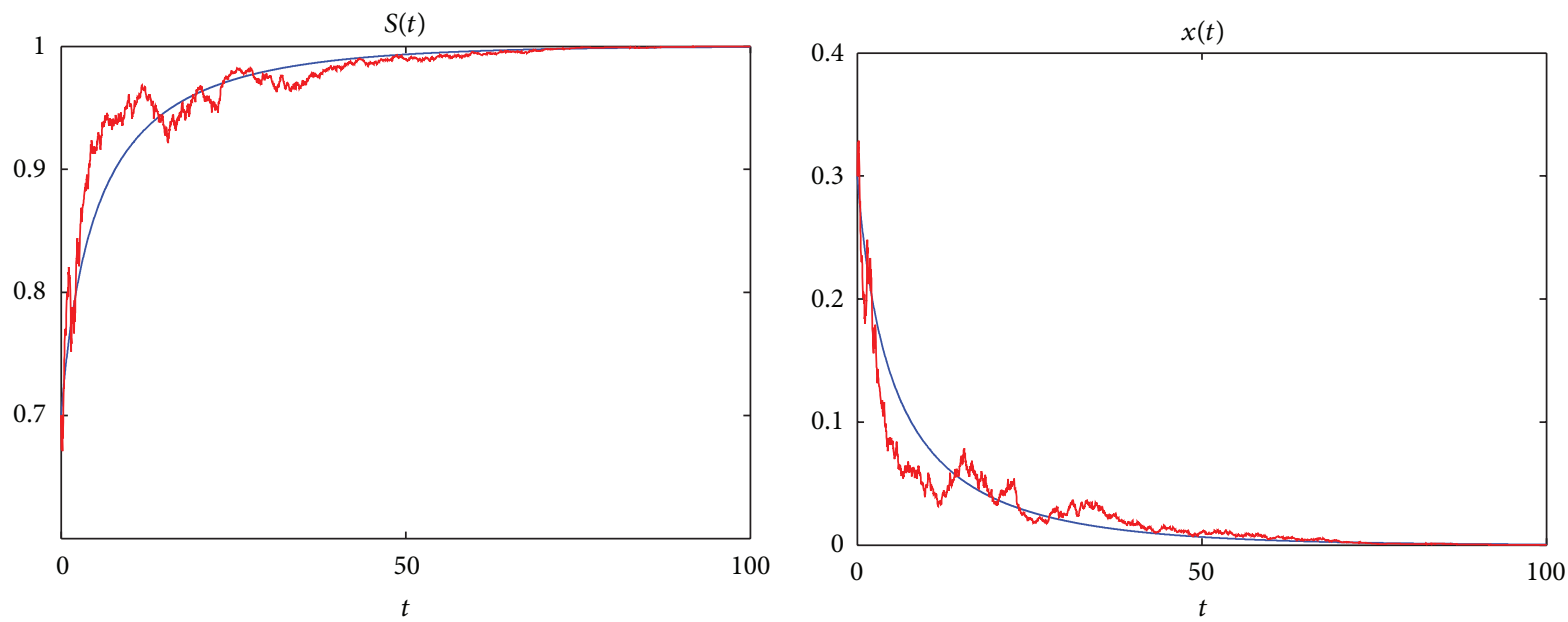

(a)
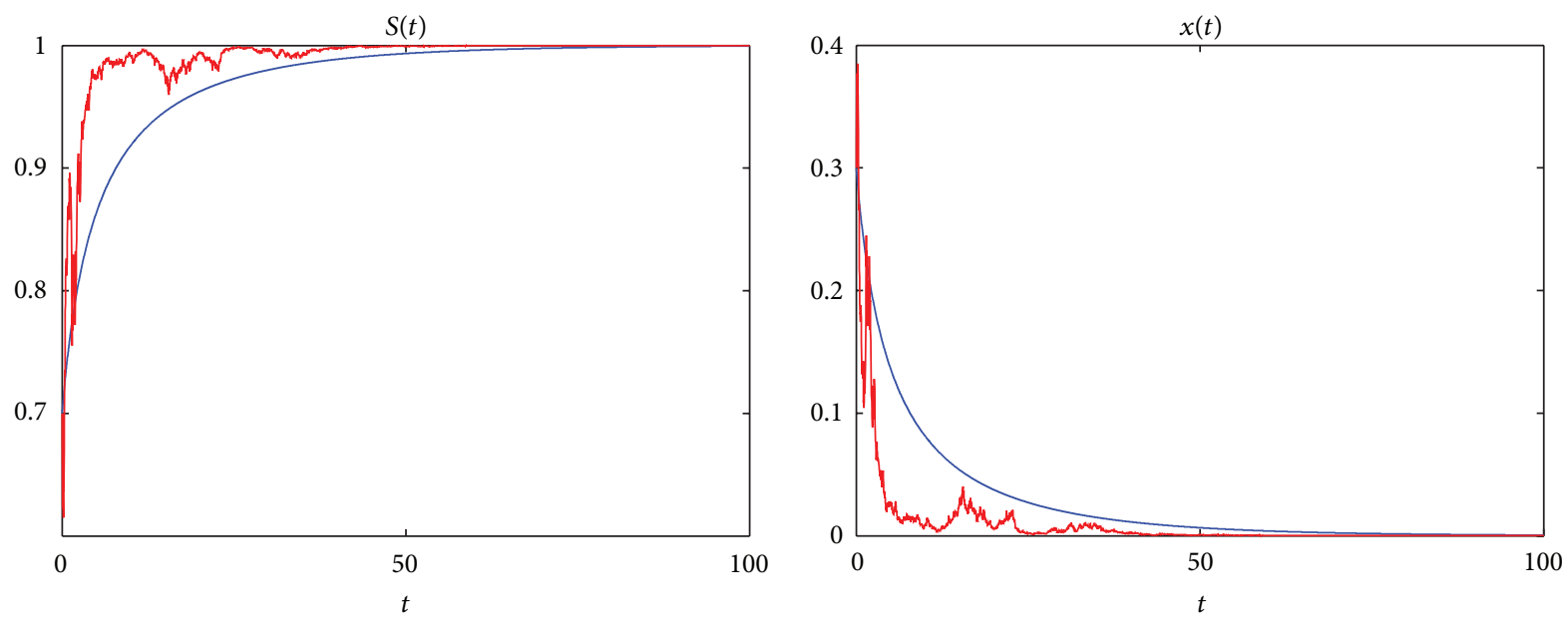

(b)

FIGURE 1: Deterministic and stochastic trajectories of simple chemostat model for initial condition $S_{0}=0.7 ; x_{0}=0.3$; and $S^{0}=1, m=2$, $a=0.6$, and $D=1.3$. (a) $\alpha=0.2$, (b) $\alpha=0.5$.

We can see that the solutions of system (3) with $\alpha=0.5$ tend to the washout equilibrium faster than that of system (3) with $\alpha=0.2$.

Next, we consider the effect of stochastic fluctuations of environment on the positive equilibrium of the corresponding deterministic system. As mentioned in the Section 2, there is a positive equilibrium $E^{*}$ of system (16) when $0<$ $\lambda<S^{0}$, and it is globally asymptotically stable. Besides, Theorem 4 tells us that the difference between the perturbed solution and $E^{*}$ is related to white noise under the conditions $0<\lambda<S^{0}$ and $\delta_{0}>0$. In systems (3) and (16), we choose parameters $S^{0}=1, m=2, a=0.6$, and $D=0.8$. As for the washout equilibrium $E^{0}$, we take $\alpha=0.1$ in Figure 2(a) and $\alpha=0.02$ in Figure 2(b). We can compute that $\lambda=0.4<1=S^{0}$ and $\delta_{0}=0.795>0$ in Figure 2(a) and $\delta_{0}=0.7998>0$ in Figure 2(b). As expected, the solutions of system (3) are oscillating around the positive equilibrium $E^{*}$ for a long time (see Figure 2). Besides, we can observe that with white noise getting weaker, the fluctuation around $E^{*}$ gets smaller, which supports the result of Theorem 4.

Theorem 8 shows that the microorganism will be washed out eventually under the condition $\alpha^{2}>2(m-D)$ even if system (16) has a positive equilibrium. As an example, we choose parameters $S^{0}=1, m=2, a=0.6$, and $D=0.8$ in systems (3) and (16). We can compute that $\lambda=0.4<$ $1=S^{0}$; thus system (16) will persist. But when $\alpha$ is large enough, for example, we take $\alpha=1.55$, we can compute that $\alpha^{2}=2.4025>2.4=2(m-D)$, and the microorganism will die out (see Figure 3). This shows that strong noise may lead to the extinction of the species in the chemostat.

To further confirm that Theorem 8 holds for a much lager class of growth rate function (i.e., Remark 9), we take $p(S)$ as the Holling IV functional response function $p(S)=(m S) /(a+$ $\left.b S+S^{2}\right)$ instead of $p(S)=m S /(a+S)$ and all parameters have the similar values as above except for $b=0.4$ in systems (3) and (16). Numerical simulations show that they have the similar dynamics as for $p(S)=m S /(a+S)$ (see Figure 4). 

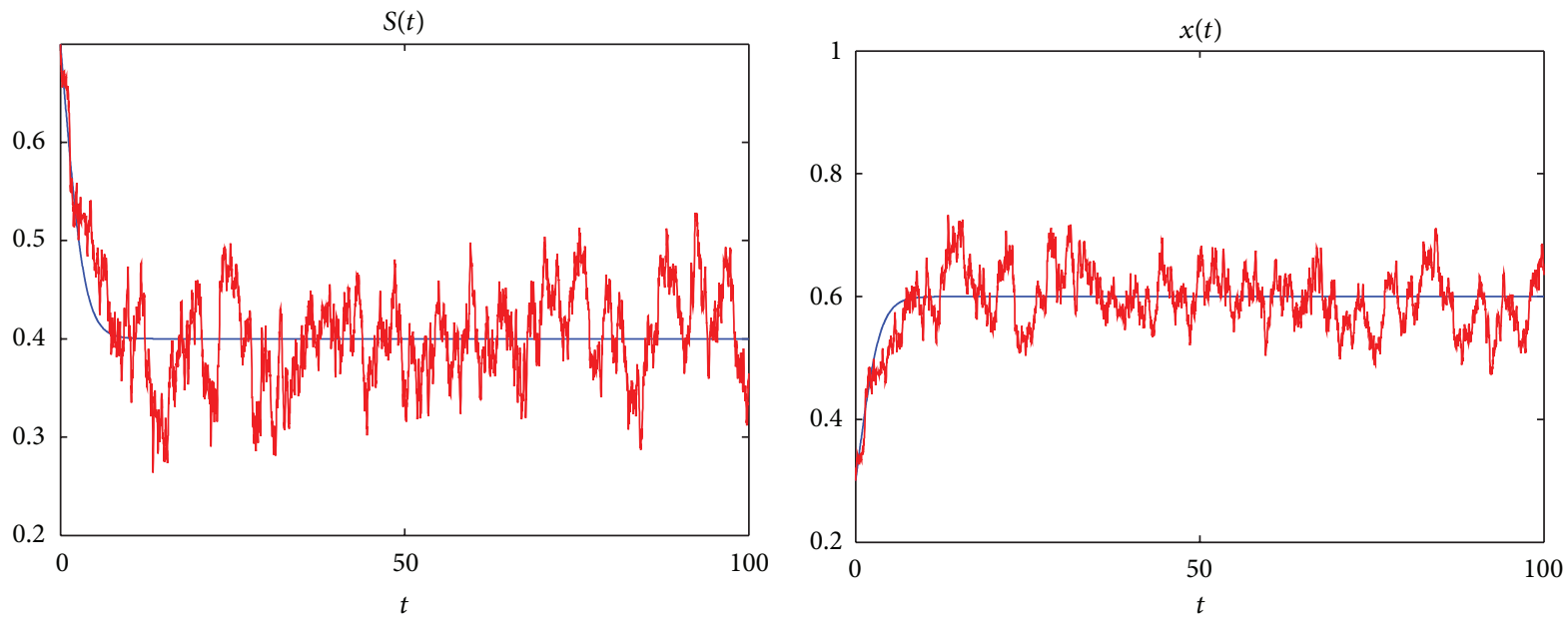

(a)
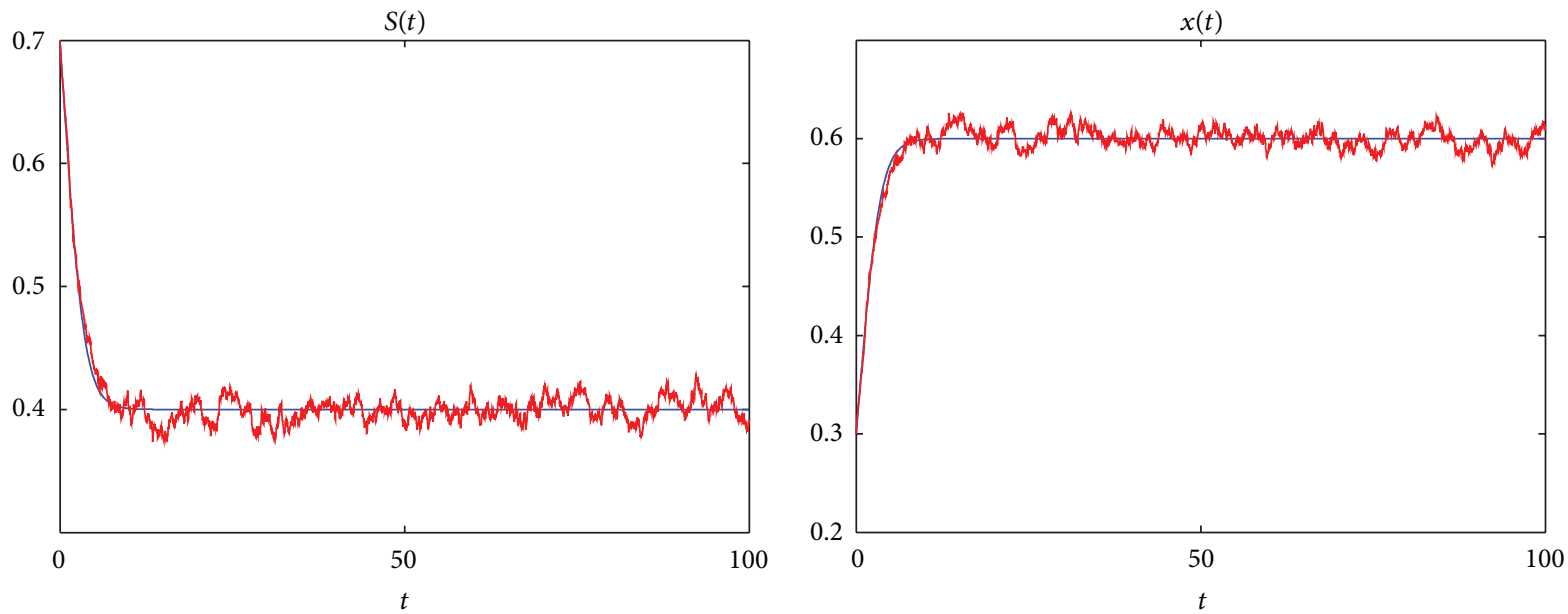

(b)

Figure 2: Deterministic and stochastic trajectories of simple chemostat model for initial condition $S_{0}=0.7 ; x_{0}=0.3$; and $S^{0}=1, m=2$, $a=0.6$, and $D=0.8$. (a) $\alpha=0.1$, (b) $\alpha=0.02$.

Some interesting questions deserve further investigation. One may propose more realistic but complex models, such as incorporating the colored noise into the system. Moreover, it is interesting to study other parameters perturbation.

\section{Appendix}

For the completeness of the paper, we list some basic theory in stochastic differential equations (see [21, 26, 27]). Let $\left(\Omega, \mathscr{F},\left\{\mathscr{F}_{t}\right\}_{t \geq 0}, P\right)$ be a complete probability space with a filtration $\left\{\mathscr{F}_{t}\right\}_{t \geq 0}$ satisfying the usual conditions (i.e., it is right continuous and $\mathscr{F}_{0}$ contains all $P$-null sets). Let $B(t)$ be the standard Brownian motions defined on this probability space. Denote

$$
\begin{aligned}
& \mathbb{R}_{+}^{n}=\left\{x \in \mathbb{R}^{n}: x_{\mathrm{i}}>0 \forall 1 \leq i \leq n\right\}, \\
& \overline{\mathbb{R}}_{+}^{n}=\left\{x \in \mathbb{R}^{n}: x_{\mathrm{i}} \geq 0 \quad \forall 1 \leq i \leq n\right\} .
\end{aligned}
$$

In general, consider $n$-dimensional stochastic differential equation

$$
d x(t)=f(x(t), t) d t+g(x(t), t) d B(t), \quad \text { on } t \geq t_{0},
$$

with initial value $x\left(t_{0}\right)=x_{0} \in \mathbb{R}^{n}$. Assume that the assumptions of the existence-and-uniqueness theorem are fulfilled. Hence, for any given initial $x_{0},(A)$ has a unique global solution that is denoted by $x\left(t ; t_{0}, x_{0}\right)$. Assume furthermore that

$$
f(0, t)=0, \quad g(0, t)=0, \quad \forall t \geq t_{0} .
$$

So $(A)$ has the solution $x(t) \equiv 0$ corresponding to the initial value $x_{0}=0$. This solution is called the trivial solution or equilibrium position.

Denote by $C^{2,1}\left(\mathbb{R}^{n} \times\left[t_{0}, \infty\right] ; \overline{\mathbb{R}}_{+}\right)$the family of all nonnegative functions $V(x, t)$ defined on $\mathbb{R}^{n} \times\left[t_{0}, \infty\right]$ such that 

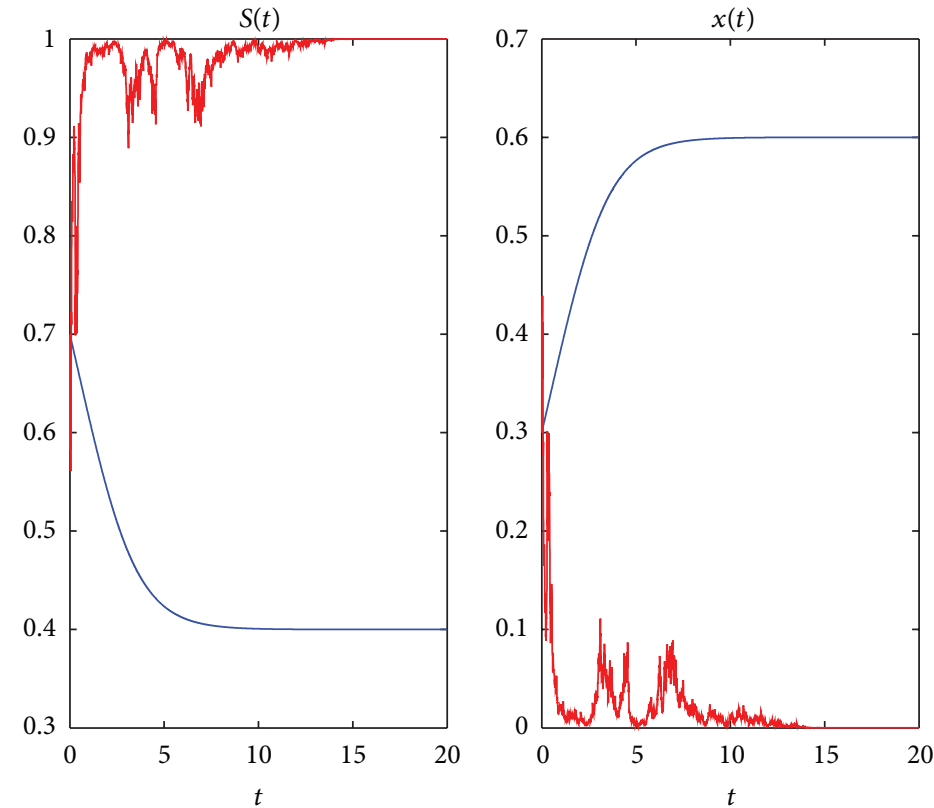

FIGURE 3: Deterministic and stochastic trajectories of simple chemostat model for initial condition $S_{0}=0.7 ; x_{0}=0.3 ;$ and $S^{0}=1, m=2$, $a=0.6, D=0.8$, and $\alpha=1.55$.
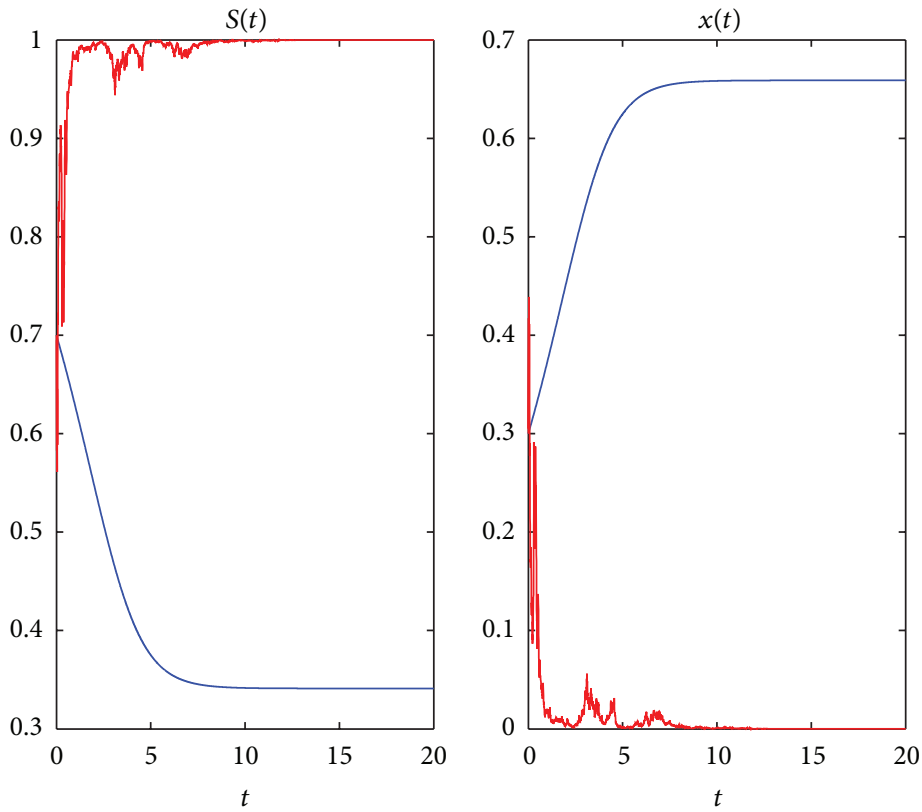

FIGURE 4: Deterministic and stochastic trajectories of chemostat model with Holling IV functional response function for initial condition $S_{0}=0.7 ; x_{0}=0.3$; and $S^{0}=1, m=2, a=0.6, b=0.4, D=0.8$, and $\alpha=1.55$.

they are continuously twice differentiable in $x$ and once in $t$. Define the differential operator $L$ associated with $(A)$ by

$L=\frac{\partial}{\partial t}+\sum_{i=1}^{n} f_{i}(x, t) \frac{\partial}{\partial x_{i}}+\frac{1}{2} \sum_{i, j=1}^{n}\left[g^{T}(x, t) g(x, t)\right]_{i j} \frac{\partial^{2}}{\partial x_{i} \partial x_{j}}$.
If $L$ acts on a function $V \in C^{2,1}\left(\mathbb{R}^{n} \times\left[t_{0}, \infty\right] ; \overline{\mathbb{R}}_{+}\right)$, then

$$
\begin{aligned}
L V(x, t)= & V_{t}(x, t)+V_{x}(x, t) f(x, t) \\
& +\frac{1}{2} \operatorname{trace}\left[g^{T}(x, t) V_{x x}(x, t) g(x, t)\right] .
\end{aligned}
$$


Definition $A$. (i) The trivial solution of $(A)$ is said to be stochastically stable or stable in probability if for every pair of $\varepsilon \in(0,1)$ and $r>1$, there exists a $\eta=\eta\left(\varepsilon, r, t_{0}\right)>0$ such that

$$
P\left\{\left|x\left(t ; t_{0}, x_{0}\right)\right|<r \forall t \geq t_{0}\right\} \geq 1-\varepsilon,
$$

whenever $\left|x_{0}\right|<\eta$. Otherwise, it is said to be stochastically unstable.

(ii) The trivial solution is said to be stochastically asymptotically stable if it is stochastically stable and, moreover, for every $\varepsilon \in(0,1)$, there exists a $\eta_{0}=\eta_{0}\left(\varepsilon, t_{0}\right)>0$ such that

$$
P\left\{\lim _{t \rightarrow \infty} x\left(t ; t_{0}, x_{0}\right)=0\right\} \geq 1-\varepsilon,
$$

whenever $\left|x_{0}\right|<\eta_{0}$.

(iii) The trivial solution is said to be stochastically asymptotically stable in the large if it is stochastically asymptotically stable and, moreover, for all $x_{0} \in \mathbb{R}^{n}$,

$$
P\left\{\lim _{t \rightarrow \infty} x\left(t ; t_{0}, x_{0}\right)=0\right\}=1 .
$$

Theorem A. If there exists a positive-definite decreasing radially unbounded function $V(x, t) \in C^{2,1}\left(\mathbb{R}^{n} \times\left[t_{0}, \infty\right] ; \overline{\mathbb{R}}_{+}\right)$, such that $\operatorname{LV}(x, t)$ is negativedefinite, then the trivial solution of $(A)$ is stochastically asymptotically stable in the large.

\section{Acknowledgments}

The authors are grateful to the handling editor and anonymous referees for their careful reading and constructive suggestions which lead to truly significant improvement of the paper. Research is supported by the National Natural Science Foundation of China (11271260, 11001212, and 1147015) and the Innovation Program of Shanghai Municipal Education Commission (13ZZ116).

\section{References}

[1] J. Monod, Recherches sur la Croissance des Cultures Bacteriennes, Herman Press, Paris, France, 1942.

[2] F. M. Stewart and B. R. Levin, "Partitioning of resources and the outcome of interspecific competition: a model and some general considerations," The American Naturalist, vol. 107, pp. 171-198, 1973.

[3] S. B. Hsu, S. Hubbell, and P. Waltman, "A mathematical theory for single-nutrient competition in continuous cultures of micro-organisms," SIAM Journal on Applied Mathematics, vol. 32, no. 2, pp. 366-383, 1977.

[4] H. L. Smith and P. Waltman, The Theory of the Chemostat, vol. 13 of Cambridge Studies in Mathematical Biology, Cambridge University Press, Cambridge, UK, 1995.

[5] T. C. Gard, "A new Liapunov function for the simple chemostat," Nonlinear Analysis: Real World Applications, vol. 3, no. 2, pp. 211-226, 2002.

[6] F. Campillo, M. Joannides, and I. Larramendy-Valverde, "Stochastic modeling of the chemostat," Ecological Modelling, vol. 222, no. 15, pp. 2676-2689, 2011.
[7] L. Imhof and S. Walcher, "Exclusion and persistence in deterministic and stochastic chemostat models," Journal of Differential Equations, vol. 217, no. 1, pp. 26-53, 2005.

[8] C. Ji, D. Jiang, and N. Shi, "Analysis of a predator-prey model with modified Leslie-Gower and Holling-type II schemes with stochastic perturbation," Journal of Mathematical Analysis and Applications, vol. 359, no. 2, pp. 482-498, 2009.

[9] J. Lv and K. Wang, "Asymptotic properties of a stochastic predator-prey system with Holling II functional response," Communications in Nonlinear Science and Numerical Simulation, vol. 16, no. 10, pp. 4037-4048, 2011.

[10] M. Liu, Q. Wu, and K. Wang, "Analysis of an improved epidemic model with stochastic disease transmission," Applied Mathematics and Computation, vol. 218, no. 19, pp. 9750-9758, 2012.

[11] X. Mao, G. Marion, and E. Renshaw, "Environmental Brownian noise suppresses explosions in population dynamics," Stochastic Processes and Their Applications, vol. 97, no. 1, pp. 95-110, 2002.

[12] X. Mao, "Stationary distribution of stochastic population systems," Systems \& Control Letters, vol. 60, no. 6, pp. 398-405, 2011.

[13] M. Carletti, "On the stability properties of a stochastic model for phage-bacteria interaction in open marine environment," Mathematical Biosciences, vol. 175, no. 2, pp. 117-131, 2002.

[14] E. Beretta, V. Kolmanovskii, and L. Shaikhet, "Stability of epidemic model with time delays influenced by stochastic perturbations," Mathematics and Computers in Simulation, vol. 45, no. 3-4, pp. 269-277, 1998, Delay systems (Lille, 1996).

[15] D. Jiang, C. Ji, N. Shi, and J. Yu, "The long time behavior of DI SIR epidemic model with stochastic perturbation," Journal of Mathematical Analysis and Applications, vol. 372, no. 1, pp. 162-180, 2010.

[16] D. Jiang, J. Yu, C. Ji, and N. Shi, "Asymptotic behavior of global positive solution to a stochastic SIR model," Mathematical and Computer Modelling, vol. 54, no. 1-2, pp. 221-232, 2011.

[17] K. S. Crump and Wan-S.C. O’Young, "Some stochastic features of bacterial constant growth apparatus," Bulletin of Mathematical Biology, vol. 41, no. 1, pp. 53-66, 1979.

[18] G. Stephanopoulos, R. Aris, and A. G. Fredrickson, "A stochastic analysis of the growth of competing microbial populations in a continuous biochemical reactor," Mathematical Biosciences, vol. 45, no. 1-2, pp. 99-135, 1979.

[19] J. Grasman, M. de Gee, and O. A. van Herwaarden, "Breakdown of a chemostat exposed to stochastic noise," Journal of Engineering Mathematics, vol. 53, no. 3-4, pp. 291-300, 2005.

[20] L. Arnold, Stochastic Differential Equations: Theory and Applications, John Wiley \& Sons, New York, NY, USA, 1974, Translated from the German.

[21] X. Mao, Stochastic Differential Equations and Applications, Horwood Publishing, Chichester, UK, 2nd edition, 2008.

[22] N. Ikeda, Stochastic Differential Equations and Diffusion Processes, North-Holland, Amsterdam, The Netherlands, 1981.

[23] L. S. Chen and J. Chen, Nonlinear Biological Dynamical System, Science Press, Beijing, China, 1993.

[24] C. Ji, D. Jiang, and N. Shi, "Multigroup SIR epidemic model with stochastic perturbation," Physica A, vol. 390, no. 10, pp. 17471762, 2011.

[25] D. J. Higham, "An algorithmic introduction to numerical simulation of stochastic differential equations," SIAM Review, vol. 43, no. 3, pp. 525-546, 2001. 
[26] R. Khasminskii, Stochastic Stability of Differential Equations, vol. 66 of Stochastic Modelling and Applied Probability, Springer, Heidelberg, Germany, 2nd edition, 2012.

[27] H. J. Kushner, Stochastic Stability and Control, vol. 33 of Mathematics in Science and Engineering, Academic Press, New York, NY, USA, 1967. 


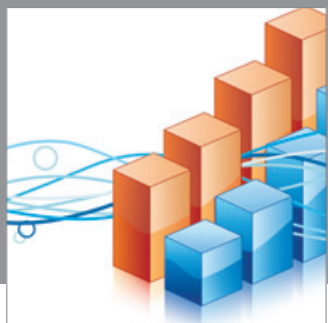

Advances in

Operations Research

mansans

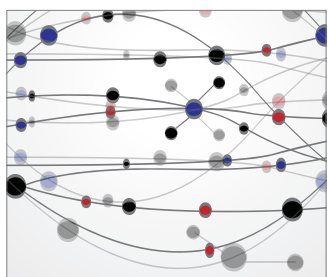

The Scientific World Journal
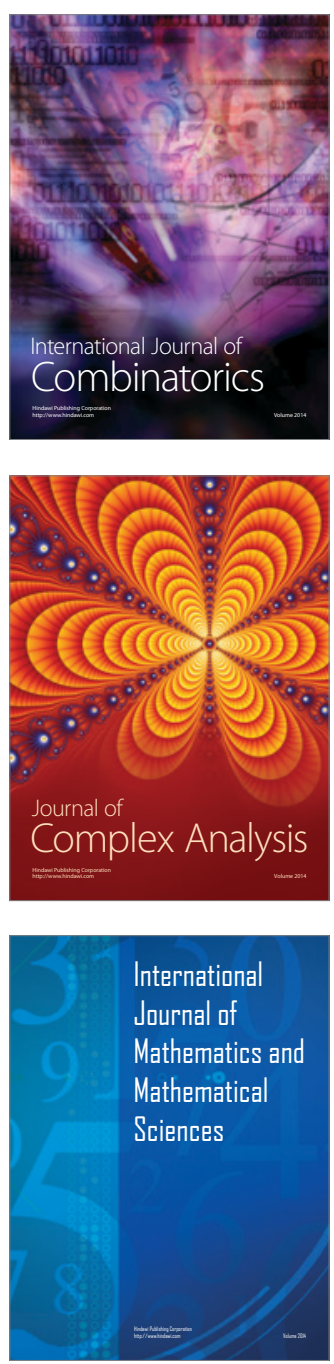
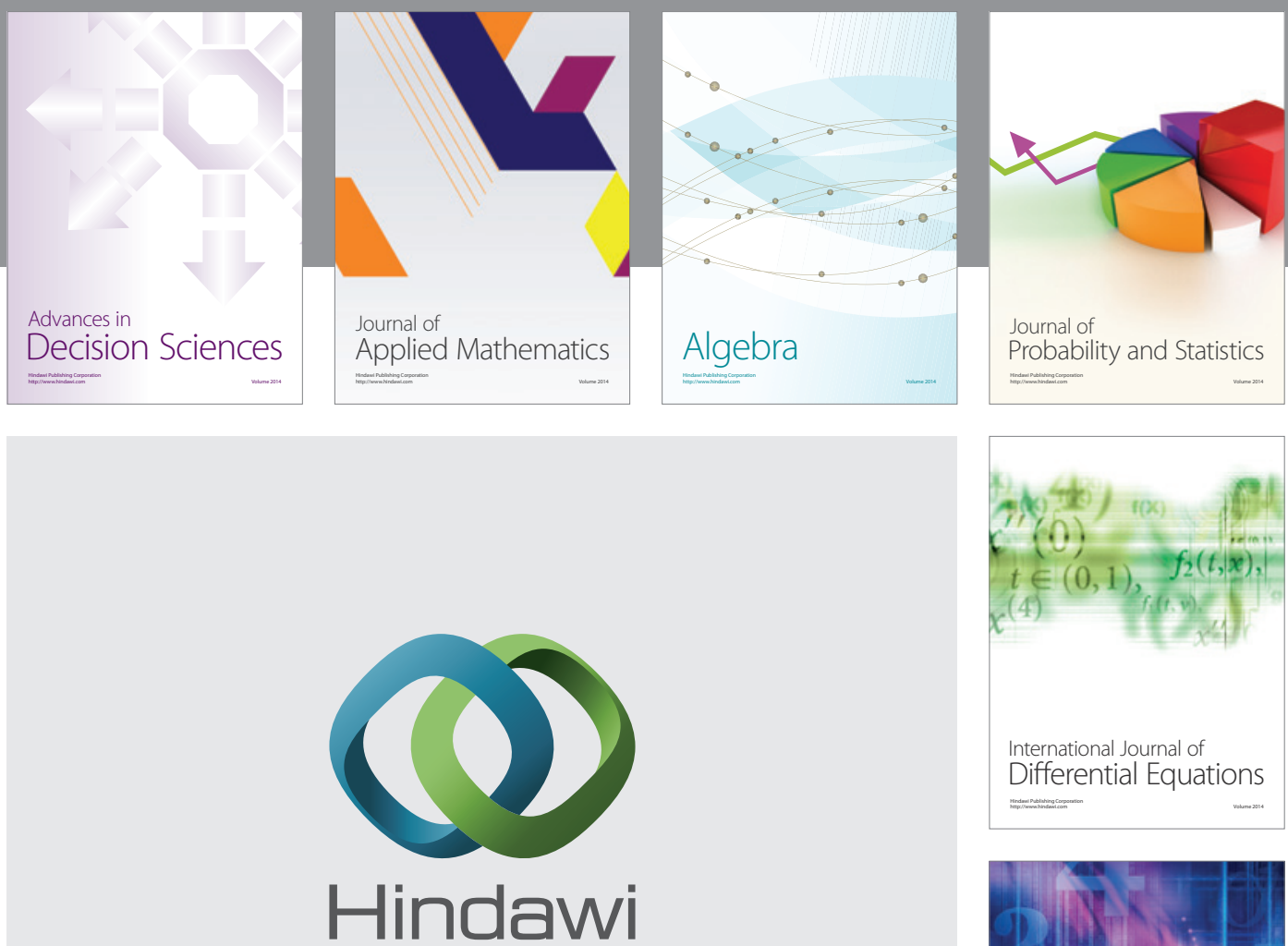

Submit your manuscripts at http://www.hindawi.com
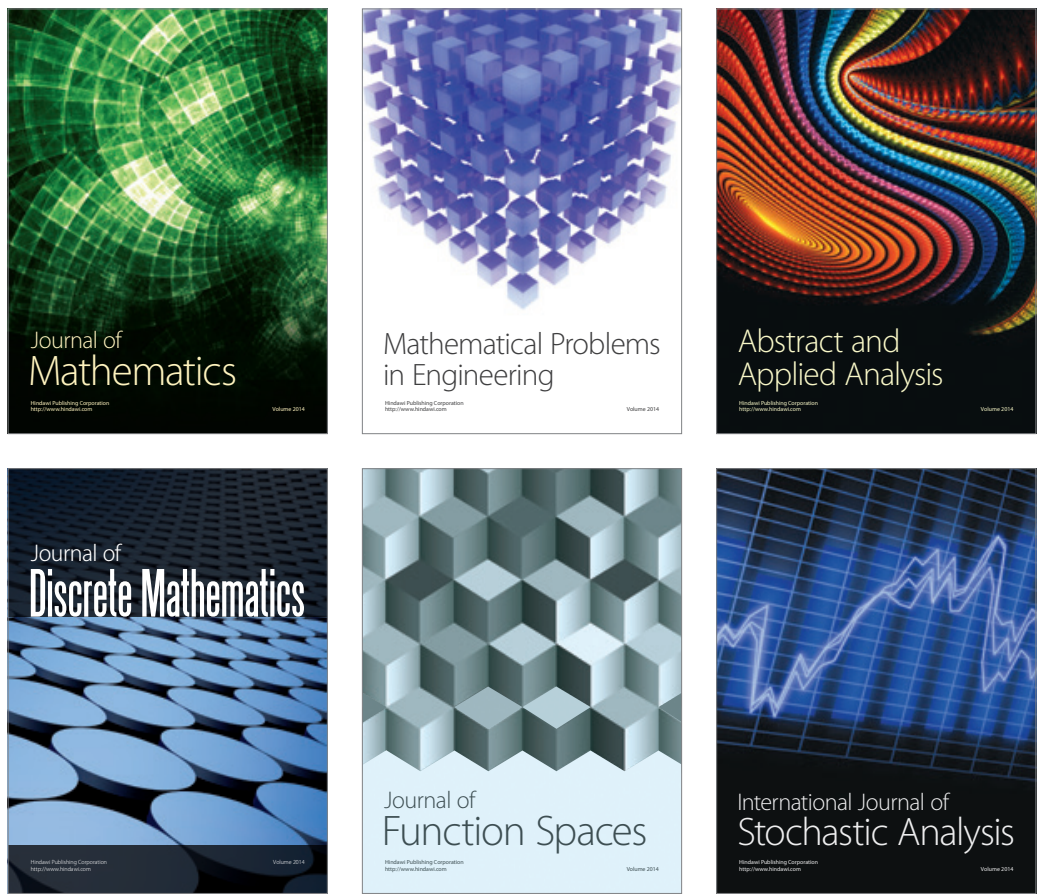

Journal of

Function Spaces

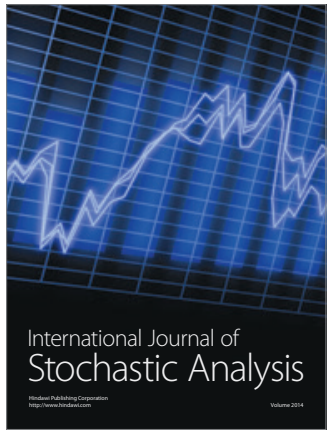

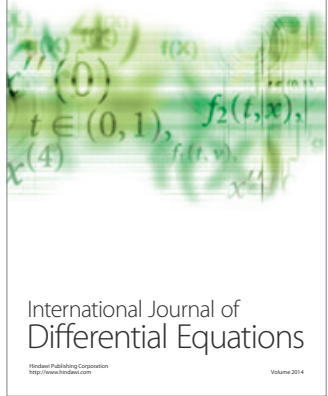
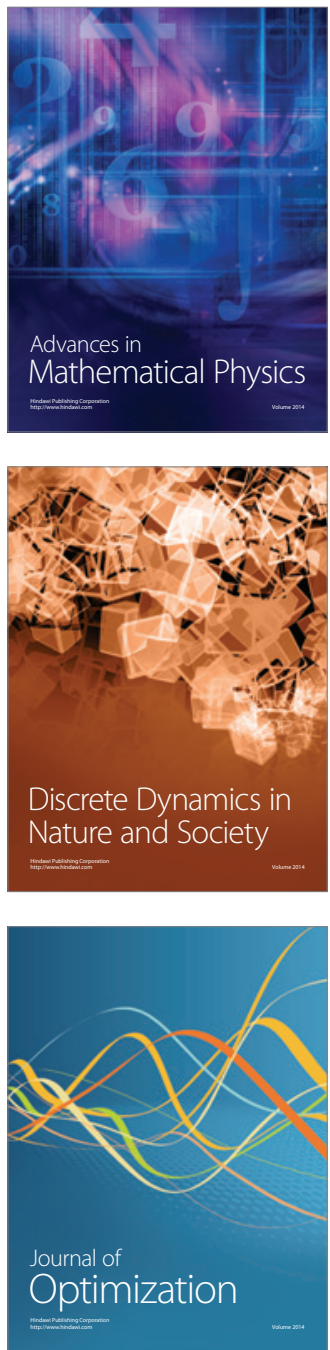